\title{
Data Processing for Time-Domain Fluorescence Lifetime Imaging Microscopy
}

\author{
Pamela A. Young ${ }^{1}$, Aivar Grislis ${ }^{1}$, Paul R. Barber ${ }^{2}$, Patricia J. Keely ${ }^{1}$, Kevin W. Eliceiri ${ }^{1}$ \\ ${ }^{1}$ Laboratory for Optical and Computational Instrumentation, University of Wisconsin, 1675 Observatory \\ Drive, 271 Animal Sciences, Madison, WI 52706-1284, United States \\ ${ }^{2}$ Gray Institute for Radiation Oncology \& Biology, Department of Oncology, University of Oxford, Old \\ Road Campus Research Building, Roosevelt Drive, Oxford OX3 7DQ
}

Fluorescence lifetime imaging microscopy (FLIM) [1] is a powerful tool for studying interactions between proteins [2] and investigating changes in the microenvironment in cells and tissues [3]. The amount of time a fluorophore resides in the excited state depends upon its microenvironment, such as $\mathrm{pH}$, hydrophobic changes, and protein interactions. FLIM is commonly used to distinguish between protein-free and protein-bound states of a molecule, and has been used to distinguish between healthy and disease state in tissue samples.

Time-domain FLIM utilizes a Time-Correlated Single Photon Counting (TCSPC) board in conjunction with a periodical light source, typically a pulsed laser, and a photon counting detector. The TCSPC electronics are capable of measuring the detection times of individual photons and reconstructing the waveform of the individual time measurements. This means each pixel in the image contains a histogram of photon counts versus time. Total number of photons in each pixel can be used to create an intensity image. However, to extract the fluorescence lifetime information, the data from each pixel must be fit to an exponential decay curve. From these fits, new images can be created using the fluorescence lifetime.

FLIM including TCSPC FLIM has seen rapid adoption by the biological microscopy community with FLIM support now commercially available for a number of different microscopy platforms ranging from widefield and confocal to multiphoton microscopes. Despite this emergence as a important modality for many studies, FLIM has not seen the corresponding increase in informatics solutions for the handling of FLIM data. Few tools exist for the sharing and analysis of FLIM data and most of those are commercial proprietary solutions.

Two of the most popular commercially available TCSPC solutions are from Becker \& Hickl [4] and PicoQuant. These companies provide software to analyze fluorescence lifetime data, SPCImage and SymPhoTime respectively. However, due to the closed "black box" aspects of these software packages, it is important to have alternative open source options available that can be used to confirm results and share data among collaborators that do not have access to these systems or use other FLIM systems. TRI2 [5] and SLIM Plugin are freely available software packages for analyzing FLIM data. TRI2 was developed by Paul Barber at the University of Oxford and gives the user many options for adjusting fitting parameters that are explicitly defined. TRI2 also has the ability to batch process images. TRI2 is powered by the open source SLIM Curve library developed in collaboration by Oxford and LOCI. SLIM Curve was created to be a full open FLIM analysis library for all curve fitting and other FLIM analysis algorithms. SLIM Curve is not only the heart of the analysis for TRI2 but also powers the SLIM Plugin for ImageJ [6]. SLIM Plugin was developed to not only fully support FLIM analysis but also integrate FLIM analysis into the other image analysis routines of ImageJ. SLIM Plugin uses Bio-Formats, [7] a Java library for reading and writing life sciences image file formats, to open proprietary FLIM data. It is 
also capable of batch processing and as a part of Image J can be used with other functions and plugins in ImageJ to allow users to do image processing with their FLIM data sets.

Advances in data processing for FLIM images have become increasingly important as this technique has become more widespread, especially among biologists who may not be experts in curve fitting and statistical analysis approaches. Making these analytical tools widely available is important to continue the growth of this technique. Future work will include support for other FLIM approaches including Frequency domain [8] and improving on support for FLIM analysis in database systems such as the Open Microscopy Environment [9].

References:

[1] JR Lakowicz, H Szmacinski, K Nowaczyk, et al, Anal Biochem, 202 (1992), p. 316-30.

[2] A Periasamy and RN Day in "Molecular Imaging: FRET Microscopy and Spectroscopy", (Oxford Univ. Press, New York).

[3] DK Bird, L Yan, KM Vrotsos, et al, Cancer Res, 65 (2005), p. 8766-73.

[4] W Becker in "The bh TSCPC Handbook", (Becker \& Hickl GmbH, Berlin).

[5] PR Barber, SM Ameer-Beg, J Gilbey, et al, J R Soc Interface 6(2009), S93-S105.

[6] CA Schneider, WS Rasband, KW Eliceiri, Nat Methods, 9(2012), p. 671-5.

[7] M Linkert, CT Rueden, C Allan, et al, J Cell Biol, 189(2010), p. 777-82.

[8] C Stringari, A Cinquin, O Cinquin, et al, PNAS, 108(2011), p. 313582-13587.

[9] JR Swedlow, Nat Cell Biol, 13 (2011).

[10] The authors acknowledge funding from DoD CDMRP BCRP Postdoctoral Fellowship Award (BC112240) and NIH grants R01-CA136590 and RC2GM092519.
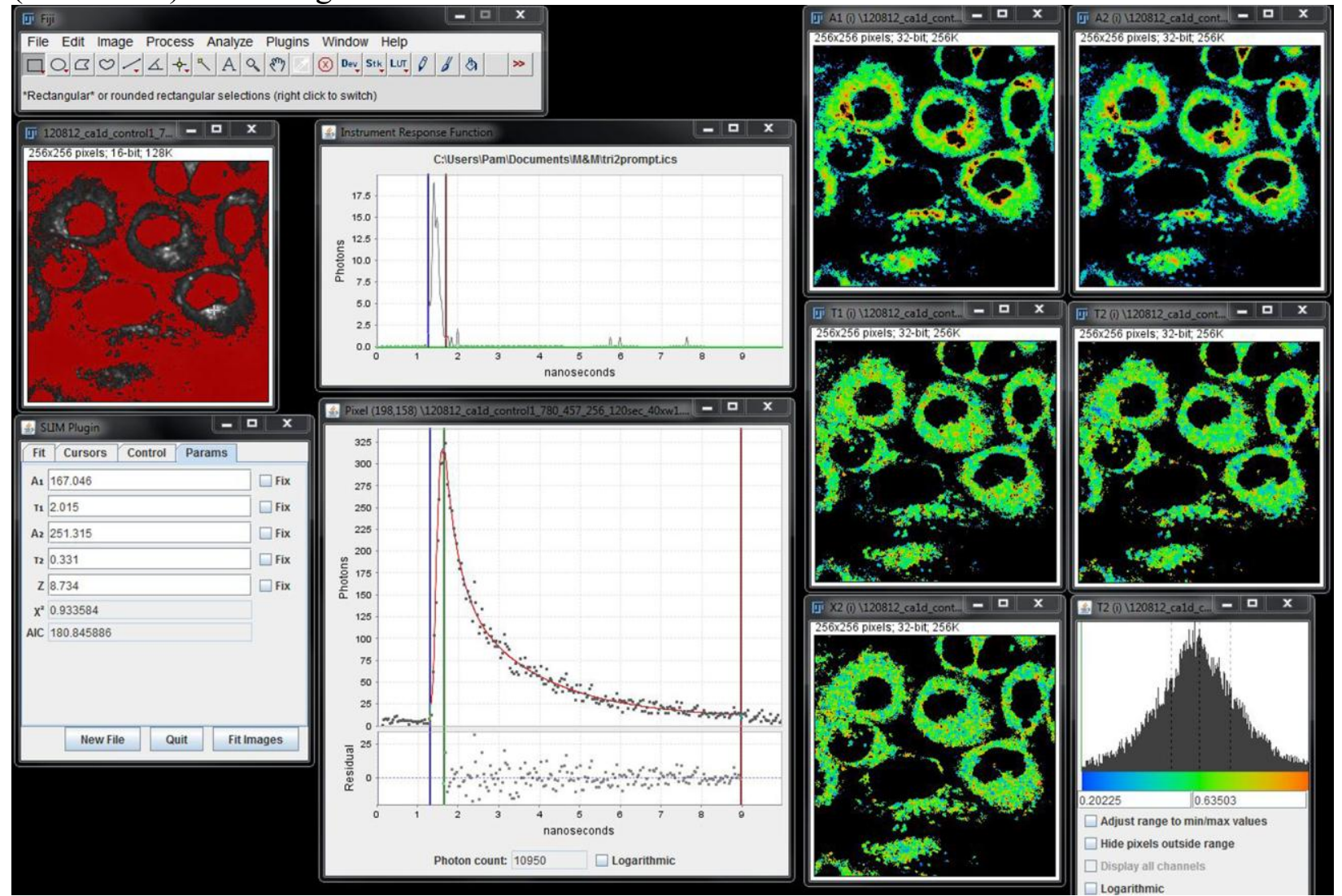

Figure 1: Lifetime analysis user interface for SLIM Plugin in ImageJ. 